\title{
Rapid Cycle Training for Non-Critical Care Physicians to Meet Intensive Care Unit Staff Shortage At An Academic Training Center In A Developing Country During the COVID-19 Pandemic
}

Abdullah Bakhsh ( $\nabla$ aarbakhsh@kau.edu.sa )

King Abdulaziz University https://orcid.org/0000-0003-1224-9367

\section{Razan Asiri}

King Abdulaziz University Faculty of Medicine

Hadeel Alotaibi

King Abdulaziz University Faculty of Medicine

Abdulaziz Boker

King Abdulaziz University

\section{Research Article}

Keywords: health simulation training, skills, competence, crisis, capacity building

Posted Date: March 1st, 2022

DOI: https://doi.org/10.21203/rs.3.rs-1295131/v1

License: (c) (i) This work is licensed under a Creative Commons Attribution 4.0 International License.

Read Full License 


\section{Abstract}

\section{Background}

The sudden unexpected increase in critically ill COVID-19 patients admitted to Intensive Care Units (ICUs), resulted in an urgent need for expanding the physician workforce. A COVID-19 Critical Care Crash Course was implemented to introduce physicians without prior critical care experience to care for critically ill COVID-19 patients. Upon successful completion of the course, physicians were recruited to work in a COVID-19 ICU under supervision of a board-certified critical care physician. The aim of this study is to describe the methods of a novel course designed specifically to teach the management critically ill COVID-19 patients, while assessing the change in knowledge, skill competency, and self-reported confidence.

\section{Methods}

The 1-day focused COVID-19 critical care crash course is composed of both virtual and practical components. Candidates may register for the practical component only after successful completion of the virtual component. We assessed knowledge acquisition using a multiple-choice question test (preand post-test assessment), skill competency, and self-reported confidence levels during simulated patient settings.

\section{Results}

Sixty-five physicians/trainees from different specialties were included in the analysis. Knowledge significantly increased from $14.92 \pm 3.20$ (out of 20 multiple-choice questions) to $18.81 \pm 1.40$ ( $p<0.01$ ), skill competence during practical stations had a mean minimum of 2 (out of 3 ), and self-reported confidence during a simulated patient setting increased significantly from $4.98 \pm 1.15$ (out of 10 ) to $8.76 \pm$ 1.10 (out of 10$)(p<0.01)$.

\section{Conclusion}

Physicians with no prior critical care experience can acquire relevant knowledge, perform sufficient basic skills and work confidently in a team after completing a focused 1-day evidence-based course, in order to increase critical care physician workforce.

\section{Background}

The COVID-19 pandemic has resulted in unparalleled strain on intensive care unit (ICU) admissions around the globe. Adequate staffing of critical care units during this time is crucial for the survival of critically ill patients. Nonetheless, the number of patient admissions to the critical care unit has exceeded the ICU workforce. Critical care training for medical personnel is not only crucial for patient survival but is also important for improving critical care team performance to standardize patient care. [1] 
As a part of COVID-19 surge planning and in anticipation for increased ICU admission, we opened an entirely new ICU dedicated for the treatment of critically ill COVID-19 patients. [2] The sudden ICU expansion resulted in even more strain on an already insufficient existing ICU medical staff resource. This resulted in an urgent need for physicians trained to manage critically ill COVID-19 patients. On the other hand, the widespread cancellation of elective surgery and outpatient appointment led to the availability of physicians/trainees from a wide range of specialties and experience levels. A study by Atagi et al. published in 2013, implemented a new Fundamentals of Critical Care Support (FCCS) course in Japan. This resulted in improved knowledge, confidence, and skills of participants with and without prior critical care experience. [3] Another study by Wanjiku at al. developed and evaluated a novel, low cost, emergency trauma course based on principles from Advanced Trauma Life Support (ATLS) but adapted to the needs of participants and resources in Kenya. Participants demonstrated statistically significant improvements in knowledge, skills and confidence in trauma management. [4]

During medical crisis and emergencies, the formula of survival encompasses three interactive elements. As key determinants of survival, these elements are medical science, education efficiency, and local implementation.[5] Educational efficiency is the instructional design of educational offerings. Education science informs how we integrate instructional design features into educational offerings in different contexts. Enhancing instructional design can improve educational outcomes, which ultimately translates to improved patient outcomes and survival. [5]

Various courses are delivered to healthcare providers from different medical backgrounds. A consistent method of teaching could harmonize different training experiences to the quest for medical knowledge and skills, especially during the challenging nature of the pandemic. [6] The purpose of this study is to assess the impact of implementing a novel COVID-19 critical care crash course on knowledge acquisition and gaining confidence while ensuring clinical skill competency for physicians and trainees without prior critical care experience.

Therefore, we undertook the task to rapidly organize a focused 1-day hands-on course to train nonintensivist physicians/trainees to care for critically ill COVID-19 patients. The purpose of the course was to teach physicians/trainees without prior critical care experience, how to participate in the management of this unique patient population. The aim of this study is to describe the methods used and immediate results of rapid capacity building in critical care physician workforce. The emphasis of the present study is to capture and describe the processes of planning, development, timely deployment and assessment tools used for similar future situations.

\section{Methods}

Setting and Study Design

As the largest tertiary hospital in the province with 1000 bed capacity, the [***] had participated in receiving many COVID-19 critically ill patients. The hospital's surge plan increased by up to 3-fold for its 25-intensive care unit (ICU) bed capacity. 
All hospital trainees and physicians were invited to attend the training program. The entire course was composed of 2 parts: 1) virtual, and 2) hands-on. The virtual part focused on introducing candidates to basic theoretical aspects on COVID-19, basic elements of critical care management, and concepts of infection control and prevention. Moreover, it included pre-recorded webinars and lectures covering guidelines and best practice strategies in managing critically III patients with suspected or confirmed COVID-19. This included basic airway management and respiratory support (initial non-invasive and invasive ventilator settings), early risk communications and inter professional teamwork. The virtual part was designed and developed by the Health Academy of the Saudi Commission for Health Specialties (SCFHS). [7] All candidates must successfully complete the virtual part before registering for the handson part of the course. Thereafter, the practical component was a focused 1-day hands-on course conducted at the $\left[{ }^{\star \star *}\right]$ - Clinical Skills and Simulation Center (KAU-CSSC).

Hand-on Course development

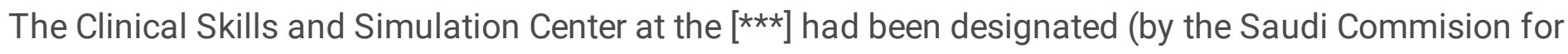
Health Specialties) as a location to conduct the hands-on part of the course. This single day focused course was developed carefully by senior anesthesiologists, emergency physicians and critical care physicians with extensive teaching experience. This program was designed to allow candidates to practice basic airway management techniques on airway manikins, to operate and change initial invasive and non-invasive mechanical ventilator settings, to practice donning and doffing of personal protective equipment, and applying theoretical knowledge in a high-fidelity simulation session (see supplementary file 1 for program agenda). Trainees and physicians from non-critical care specialties who completed the online part were eligible to register for the hands-on part. Physical distancing and strict hygiene precautions were implemented for participant and instructor safety.

Instructors were board-certified physicians in anasthesia, critical care and emergency medicine. Additionally, respiratory practitioners and nursing educators were carefully recruited as instructors. Moreover, course director and instructors were certified in item writing analysis and train the trainer program. The course director ensured standardization of course content among instructors to meet candidate needs. Moreover, supplementary ID-sized cards were created and distributed to candidates as cognitive aids for use at the bedside (supplementary file 2 and 3 ). These cards were attached to badge holders for speedy access (as a cognitive aid) and help candidates make the right decisions quickly when it comes to critical medication dosage, initial mechanical ventilator settings, and personal protective equipment sequencing.

Knowledge Assessment tool

A multiple-choice question test was developed by a group of active clinical faculty from anasthesia, critical care and emergency medicine with expertise in education and testing. Multiple choice questions (MCQs) were developed following best practices for construction and phrasing. The MCQs tested knowledge on basic airway management, the use of personal protective equipment, initial mechanical ventilator settings, vasopressors and hemodynamic parameters, and team dynamics. Four faculty 
members met to decide on items relevant for the purpose of the program. Consensus was reached on 20 MCQs after two iterations. Each question had one best answer and three wrong answers. The surviving sepsis campaign guidelines on the management of adults with coronavirus disease 2019 in the ICU were used as the primary reference for writing MCQs. The pre-test was distributed and completed electronically via Google form on the day of the course prior to starting. While the post-test was distributed and completed immediately after course completion.

Competency assessment

A competency checklist was developed by the Saudi Commission for Health Specialties (SCFHS) for each hands-on station. Residents and physicians participating in the course are expected to demonstrate basic skills. The purpose of these hands-on stations is to assess candidate competency (supplementary file 3 ) and re-enforce skills, while ensuring candidates ability to perform independently, while awaiting for more advanced help in real clinical practice.

Performance assessment Tool

A single simulation-based scenario was case implemented using a high-fidelity manikin in a simulated environment (supplementary file 4). This scenario was developed by the SCFHS and reviewed by the site course director and instructors at the clinical skills and simulation center. The scenario was modified and adopted to our institution's established diagnostic and therapeutic protocols. Candidates self-reported confidence levels (on a scale of 0-10) were used to assess candidate confidence before and after simulation-based scenario.

\section{Statistical analysis}

Summary statistics and skill competency were performed using frequencies and means, where appropriate. Pre- and post-training tests, and self-reported confidence levels were compared using paired T-test. A $p<0.05$ was considered as statistically significant.

\section{Results}

One-hundred and thirteen physicians/trainees participated in the course. This was a 1-day focused course lasting 5-hours. A total of 7 courses were completed in June and July 2020; preparing 113 physicians to add to the physician workforce. We included a total of 65 participants in the analysis. Fortyeight participants were excluded for not consenting to participate in the study. Thirty-five (53.8\%) participants were male whereas 58 (89.2\%) were trainees, while only 7 participants were board-certified physicians. Table 1 shows participant characteristics. 
Table 1

Participant characteristics

\begin{tabular}{|ll|}
\hline Gender & N (\%) \\
\hline Male & $35(53.8 \%)$ \\
\hline Female & $30(46.2 \%)$ \\
\hline Specialty & \\
\hline Anasthesia & $7(10.8 \%)$ \\
\hline Critical care medicine & $3(4.6 \%)$ \\
\hline Emergency medicine & $1(1.5 \%)$ \\
\hline General surgery/surgical subspecialties & $4(6.1 \%)$ \\
\hline Internal medicine & $45(69.2 \%)$ \\
\hline Pediatrics & $3(4.6 \%)$ \\
\hline Obstetrics and gynecology & $2(3.1 \%)$ \\
\hline Level & \\
\hline Trainee & $58(89.2 \%)$ \\
\hline Physician & $7(10.7 \%)$ \\
\hline
\end{tabular}

The mean pre-course test score was 14.92 points (out of 20 points). The post-course test immediately after the course score significantly increased to 18.81 points (out of 20$)(p<0.01)$. Participants selfreported confidence rate significantly increased from 4.98 (out of 10) before simulation-based scenario to 8.76 (out of 10 ) after simulation-based scenario $(p<0.01)$. Furthermore, candidates mean competency scores were 2.80, 2.33, and 2.03 (out of 3 ) during the personal protective equipment station, basic airway station, and mechanical ventilator station, respectively. Table 2 describes participants knowledge acquisition, competency, and self-reported confidence level. 
Table 2

Participant's knowledge acquisition, competency, and self-reported confidence level

\begin{tabular}{|c|c|c|}
\hline Pre/post-course assessment (out of 20) & Mean \pm (SD) & p-value \\
\hline Pre-course assessment score & $14.92 \pm 3.20$ & \multirow[t]{2}{*}{$P<0.01$} \\
\hline Post-course assessment score & $18.81 \pm 1.40$ & \\
\hline Station competency (out of 3 ) & & \multirow[t]{5}{*}{ - } \\
\hline Personal protective equipment & $2.80 \pm 0.40$ & \\
\hline Basic airway & $2.33 \pm 0.47$ & \\
\hline Mechanical ventilation & $2.03 \pm 0.66$ & \\
\hline Self-reported confidence level (out of 10) & & \\
\hline Pre-course self-reported confidence level & $4.98 \pm 1.15$ & \multirow[t]{2}{*}{$P<0.01$} \\
\hline Post-course self-reported confidence level & $8.76 \pm 1.10$ & \\
\hline
\end{tabular}

\section{Discussion}

Our findings reveal that this course effectively facilitated knowledge acquisition and improved selfreported confidence, while ensuring basic clinical skill competency. These effects were seen in a wide variety of candidates including both trainees and physicians without critical care experience. The COVID19 critical care crash course offered some advantages during the pandemic. First, the theoretical part is provided through a virtual learning platform, limiting close contact. Second, the practical part is short and no longer than 5-hours. Moreover, the teaching guide and practical stations are focused and succinct. These qualities are convenient for learners and educators amid a busy schedule, while rapidly increasing the ICU workforce.

COVID-19 pandemic has shown healthcare systems vulnerabilities at many levels, particularly in terms of supply chains disruption, accessibility, and human resources. [8] Having a resilient system require attention to all these elements. Critical care staffing in of itself is a challenge during routine care; the progressive increase in demand with the outbreak was expected to stress an already strained situation. [9, 10] Having a quick deployable means to bridge the expected gap have been done using variety of methods worldwide. [11]

Previous studies have demonstrated increased knowledge acquisition via pre- and post-course assessment scores in advanced trauma life support, advanced cardiac life support and fundamentals of critical care. $[12,13,14,15,16]$ Our course was designed to introduce physicians with no prior critical care experience to work in critical care units designated for COVID-19 patients. The results of our study showed an increase in basic knowledge via an internally validated 20-mulitple choice question (MCQ) test. Multiple choice questions are easily scalable and require little time and few resources. The test can 
be completed in approximately 20-minutes, and by using an online version, test results can be readily available. Therefore, it is repeatable with minimal cost. A weakness of multiple-choice questions is that it does not allow for qualified elaborate answers. Most importantly, MCQ format only test knowledge. Furthermore, all candidates scored at least a mean of 2 out of 3 during skill stations. This indicates all candidates performed skills sufficiently but need further practice, which is expected. Skill stations were designed to assess and reinforce selected skills, which are expected from non-critical care physicians and trainees. Additionally, self-reported confidence levels increased significantly during performance-based training in a simulated patient setting. Simulation-based training has well documented benefits and is an efficient way of providing training in a safe and protected environment. However, simulation-based training is resource intensive.

This study demonstrates that it is possible to develop a course focusing on knowledge, skills, and team performance despite a time-limited situation. Our findings and course materials are important resources to be used for similar situations in the future or during resurge of cases.

\section{Limitations}

Our study did not measure actual clinical performance. Neither did this study look at long-term knowledge retention or patient centered outcomes when graduates of this course staff critical care units. Although, we included 65 participants in the analysis, this sample size is insufficient to explore differences between specialties.

\section{Conclusion}

In this study, we developed a focused 1-day course for trainees and physicians without prior critical care experience. We found this course extremely valuable to prepare trainees and physicians volunteering to participate in managing critically ill COVID-19 patients, as it significantly increased knowledge and selfreported confidence in approaching critically ill COVID-19 patients.

\section{Abbreviations}

ATLS: advanced trauma life support

COVID-19: coronavirus disease 2019

FCCS: fundamentals of critical care support

ICU: intensive care unit

ID: identification

KAU-CSSC: king abdulaziz university-clinical skills and simulation center 
MCQ: multiple choice question

\section{Declarations}

\section{Ethics approval and consent to participate}

The unit of biomedical ethics at the King Abdulaziz University has exempted our study from approval (reference 03-21). Written consent was obtained from all participants at the beginning of the course.

\section{Consent for publication}

Not applicable

\section{Availability of data and materials}

The dataset analyzed in the current study is available as a supplementary Excel file (data file). Educational materials are publicly made available as supplementary files. Multiple choice questions (MCQs) are available upon reasonable request from the corresponding author.

\section{Competing interests}

The authors declare that they have no competing interests

\section{Funding}

Not applicable

\section{Authors' contributions}

$A A$ and $A B$ designed the study; RA and $H A$ conducted the research; $A B$ and RA analyzed the data; $A A, A B$, and $\mathrm{HA}$ wrote the manuscript; and $A B$ had primary responsibility for the final content. All authors read and approved the final manuscript

\section{Acknowledgements}

The authors express their gratitude toward the technicians and administrative staff at the king abdulaziz university - clinical skills and simulation center for their diligent work in making this endeavor a success.

\section{References}

1. Sevdalis N, Brett SJ. Improving care by understanding the way we work: human factors and behavioural science in the context of intensive care. Crit Care. 2009;13:139. 10.1186/cc7787.

2. Farsi S, Noaman N, Bukhary A, et al. Anaesthesia and Critical Care Department at a Major Academic Centre's Adaptation to Face the COVID-19 Pandemic. Int J Gen Med. 2021;15:14:3539-52. $10.2147 /$ IJGM.S318336. 
3. Atagi, et al. Evaluating the fundamental critical care support course in critical care education in Japan: a survey of Japanese fundamental critical care support course experience. 2013. 1;1(1):5. 10.1186/2052-0492-1-5.

4. Wanjiku, et al. Assessing the impact of an emergency trauma course for senior medical students in Kenya. 2017. 7(4); 167-171. 10.1016/j.afjem.2017.04.013.

5. Cheng A, Nadkarni VM, Mancini MB, et al, on behalf of the American Heart Association Education Science Investigators; and on behalf of the American Heart Association Education Science and Programs Committee, Council on Cardiopulmonary. Critical Care, Perioperative and Resuscitation; Council on Cardiovascular and Stroke Nursing; and Council on Quality of Care and Outcomes Research. Resuscitation education science: educational strategies to improve outcomes from cardiac arrest: a scientific statement from the American Heart Association. Circulation. 2018;138:e82-122. 10.1161/CIR.0000000000000583.

6. Oddiri U, Chong G. Pediatric intensive care unit resident educational curriculum. MedEdPORTAL. 2020;16:10999. 10.15766/mep_2374-8265.10999.

7. https://.

8. Govindan K, Mina H, Alavi B. A decision support system for demand management in healthcare supply chains considering the epidemic outbreaks: A case study of coronavirus disease 2019 (COVID-19). 2020. 138:101967. 10.1016/j.tre.2020.101967.

9. Coughlan C, Nafde C, Khodatars S, et al. COVID-19: lessons for junior doctors redeployed to critical care. Postgrad Med J. 2021 Mar;97(1145):188-91. 10.1136/postgradmedj-2020-138100.

10. Haldane V, De Foo C, Abdalla SM, et al. Health systems resilience in managing the COVID-19 pandemic: lessons from 28 countries. Nat Med. 2021 Jun;27(6):964-80. 10.1038/s41591-02101381-y.

11. Lee CCM, Thampi S, Lewin B, et al. Battling COVID-19: critical care and peri-operative healthcare resource management strategies in a tertiary academic medical centre in Singapore. Anaesthesia. 2020 Jul;75(7):861-71. 10.1111/anae.15074.

12. Alsolamy S, Cluntun A, Aldekhyl S, et al. A National Initiative: Training Nonintensivists in Critical Care, an Educational Response to the COVID-19 Pandemic. Saudi Crit Care J. 2020;4(Suppl S1):34-9. 10.4103/sccj.sccj_50_20.

13. Mohammad A, Branicki F, Abu-Zidan FM. Educational and clinical impact of Advanced Trauma Life Support (ATLS) courses: a systematic review. World J Surg. 2014 Feb;38(2):322-9. 10.1007/s00268013-2294-0.

14. Ali I, Cohen R, Reznick R. Demonstration of acquisition of trauma management skills by senior medical students completing the ATLS Program. J Trauma. 1995 May;38(5):687-91.

10.1097/00005373-199505000-00002.

15. Langdorf MI, Strom SL, Yang L, Canales C, Anderson CL, Amin A, Lotfipour S. High-fidelity simulation enhances ACLS training. Teach Learn Med. 2014;26(3):266-73. 10.1080/10401334.2014.910466. 
16. Cox CE, Carson SS, Ely EW, et al. Effectiveness of medical resident education in mechanical ventilation. Am J Respir Crit Care Med. 2003 Jan 1;167(1):32-8. 10.1164/rccm.200206-6240C.

\section{Supplementary Files}

This is a list of supplementary files associated with this preprint. Click to download.

- SupplementaryFile1.pdf

- Supplementaryfile2.pdf

- Supplementaryfile3.pdf

- Supplementaryfile4.pdf 